Article

\title{
Transesophageal Echocardiography as a Monitoring Tool during Transvenous Lead Extraction-Does It Improve Procedure Effectiveness?
}

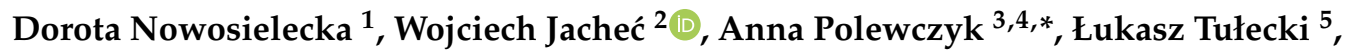 \\ Konrad Tomków ${ }^{5}$, Paweł Stefańczyk ${ }^{1}$, Andrzej Tomaszewski ${ }^{6}$, Wojciech Brzozowski ${ }^{6}$,

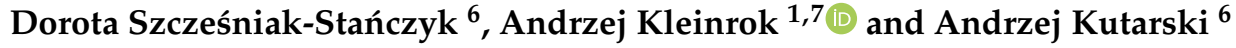 \\ 1 Department of Cardiology, The Pope John Paul II Province Hospital, 22-400 Zamosc, Poland; \\ dornowos@wp.pl (D.N.); paolost@interia.pl (P.S.); a.kleinrok@wp.pl (A.K.) \\ 2 2nd Department of Cardiology, Silesian Medical University, 41-800 Zabrze, Poland; wjachec@interia.pl \\ 3 Department of Physiology, Patophysiology and Clinical Immunology, Collegium Medicum of Jan \\ Kochanowski University in Kielce, 25-317 Kielce, Poland \\ 4 Department of Cardiac Surgery, Swietokrzyskie Cardiology Center, 25-736 Kielce, Poland \\ 5 Department of Cardiac Surgery, The Pope John Paul II Province Hospital, 22-400 Zamosc, Poland; \\ luke27@poczta.onet.pl (Ł.T.); konradtomkow@wp.pl (K.T.) \\ 6 Department of Cardiology, Medical University, 20-400 Lublin, Poland; benecho2008@gmail.com (A.T.); \\ brzozo@wp.pl (W.B.); dorotasstanczyk@gmail.com (D.S.-S.); a_kutarski@yahoo.com (A.K.) \\ 7 University of Information Technology and Management, 35-959 Rzeszow, Poland \\ * Correspondence: annapolewczyk@wp.pl; Tel.: +484-13671508; Fax: +48-600024074
}

Received: 4 April 2020; Accepted: 2 May 2020; Published: 8 May 2020

\begin{abstract}
Background: Transesophageal echocardiography (TEE) is a valuable tool for monitoring the patient during transvenous lead extraction (TLE), but the direct impact of TEE on the effectiveness and safety of TLE has not yet been documented. Methods: The effectiveness of TLE and short-term survival were compared between two groups of patients: 2106 patients in whom TEE was performed before and after TLE and 1079 individuals in whom continuous TEE monitoring was used. The procedure-related risk of major complications was assessed using a predictive SAFeTY TLE score. Results: The patients monitored by TEE were characterized by older age, more comorbidities and higher SAFeTY TLE scores $(6.143 \pm 4.395$ vs. $5.593 \pm 4.127 ; p=0.004)$. Complete procedural success was significantly higher in the TEE-guided group $(97.683 \%$ vs. $95.442 \%, p<0.01)$. The rate of serious complications in the TEE-guided group was lower than the predictive SAFeTY TLE score-a reduction of $28.75 \%$ $(p<0.05)$. Periprocedural mortality in the TEE-guided and non-TEE-guided groups was zero vs. six deaths $(p=0.186)$. Short-term survival was comparable between the groups. Conclusions: Transesophageal echocardiography as a monitoring tool during transvenous lead extraction provides valuable results-higher rates of complete procedural success and a reduced risk of the most severe complications, thus preventing periprocedural deaths.
\end{abstract}

Keywords: transesophageal echocardiography; continuous intraprocedural monitoring; transvenous lead extraction

\section{Introduction}

Transvenous lead extraction (TLE), as a method for managing complications related to cardiac implantable electronic devices (CIED), has been evolving since the 1990s and is now widely used in hospitals all over the world. The European Heart Rhythm Association (EHRA) report indicates that more than 9000 TLEs are performed annually at over 350 centers [1-3]. 
Lead extraction techniques involve manual traction or laser energy to dissect and remove lead from the cardiovascular system. Given the potential for serious complications related to cardiac tear or venous injury, it is crucial to constantly improve the safety of the procedure being performed. TLE belongs to relatively safe invasive procedures - the percentage of major complications range from 0.9 to $4.0 \%$, unfortunately, procedural deaths (up to $1.86 \%$ ) are still reported [4-14].

Both European and American guidelines published in 2017 provided more refined recommendations regarding the safety of TLE procedures. It is therefore recommended to perform TLE in an operating room or a hybrid operating room, preferably under general anesthesia, with direct systemic blood pressure monitoring via an indwelling arterial catheter, with a cardiothoracic surgeon available and with the continuous monitoring of the patient using transesophageal echocardiography (TEE) or intracardiac echocardiography (ICE) $[13,14]$. This study therefore sets out to assess the impact of continuous TEE monitoring on the effectiveness and safety of TLE. In order to estimate TLE-related risk, we use a previously devised SAFeTY TLE score to determine the probability of developing major complications during the procedure [15].

\section{Methods}

\subsection{Study Population}

This post-hoc analysis used clinical data of 3185 patients who underwent transvenous lead extraction between March 2006 and January 2020. Between the years 2006 and 2015, there were 2106 lead extractions with concomitant transthoracic echocardiography (TTE) and transesophageal echocardiography before and after the procedure, without intraprocedural monitoring. Between June 2015 and January 2020 there were 1079 lead extractions entirely under TEE guidance. All the procedures were performed at two different centers by the same experienced operator in both cases.

\subsection{Lead Extraction Procedure}

Lead extraction procedures were performed using mechanical systems such as polypropylene Byrd dilator sheaths (Cook ${ }^{\circledR}$ Medical, Leechburg, PA, USA), mainly via the subclavian approach on the side of the implanted device. If technical difficulties arose, a different vascular access and/or additional tools such as Evolution (Cook ${ }^{\circledR}$ Medical, Bloomington, IN, USA), TightRail (Spectranetix Colorado Springs, CO, USA), lassos and basket catheters were utilized. Laser cutting sheaths were not used. In the non-TEE-guided group, extraction procedures were performed by an experienced TLE operator and qualified nurses in an electrophysiology lab, in patients under general anesthesia and sedation. In the TEE-guided group lead extractions were performed by a team consisting of the same experienced TLE operator, with a second operator who had experience with pacing therapy, acardiac surgeon, anesthesiologist and echocardiographist. Procedures were performed in an operating room or in a hybrid operating room, in patients under general anesthesia, with continuous invasive blood pressure monitoring. Indications for TLE and type of periprocedural complications were defined according to the 2017 HRS Expert Consensus Statement on Cardiovascular Implantable Electronic Device Lead Management and Extraction as well as the European Lead Extraction ConTRolled (ELECTRa) study: a European Heart Rhythm Association (EHRA) Registry of Transvenous Lead Extraction Outcomes [13,14].

\subsection{Transesophageal Echocardiographic Monitoring}

Transesophageal echocardiographic monitoring was performed using the GE Vivid 3, Vivid 4, and Vivid S 70 systems, as well as the Philips iE33 ultrasound machine equipped with transesophageal probes for $3 \mathrm{D}$ and $4 \mathrm{D}$ imaging. All images were stored in a digital memory for extended analysis after the procedure. Leads were assessed in standard esophageal and transgastric views. Standard views included mid-esophageal views $\left(0^{\circ}, 30^{\circ}-35^{\circ}, 60^{\circ}-75^{\circ}, 80^{\circ}-100^{\circ}, 100^{\circ}-150^{\circ}\right)$ visualizing the right atrium, tricuspid valve, right ventricular outflow tract (RVOT) and right ventricular inflow tract 
(RVIT); low esophageal views $\left(0^{\circ}, 45^{\circ}-55^{\circ}, 80^{\circ}-90^{\circ}\right)$ visualizing the coronary sinus, tricuspid valve; and transgastric right ventricular views $\left(30^{\circ}-40^{\circ}, 90^{\circ}, 100^{\circ}-110^{\circ}, 110^{\circ}-130^{\circ}\right)$ visualizing three leaflets of the tricuspid valve, right ventricular long axis, RVOT, and right ventricular cross-sections. Sometimes, if necessary, nonconventional imaging planes were used for better visualization of the structures.

TEE monitoring was conducted in three phases: preprocedural, intraprocedural and postprocedural. The study started after patient intubation with the insertion of an echocardiographic probe down the esophagus. All study phases were performed during the same extraction procedure. In the preprocedural phase, we evaluated the lead position, degree of fibrous encapsulation, lead-to-lead adhesions, unaccounted-for structures in the leads, as well as the function of the tricuspid valve and the pericardium. The intraprocedural phase was performed during the extraction procedure, taking into account direct pulling on the cardiac walls, obliterating the right ventricular lumina and their effect on the hemodynamic status of the patient. Furthermore, we observed pulling on other leads, detachment and dislodgement of fibrous tissue or vegetation fragments, and the separation of pericardial layers. The postprocedural phase included an evaluation of tricuspid valve function, remnants of masses removed during TLE (vegetations, fibrous tissue), and the abnormal accumulation of fluid in the pericardial sac.

\subsection{Estimating the Number of Major Complications Using the SAFeTY TLE Score}

The SAFeTY TLE score assesses the risk of the occurrence of major complications related to TLE and takes into account the following parameters: sum of dwell times of extracted leads (threshold value $\geq 16.5$ years), hemoglobin level in the blood (threshold level $<11.5 \mathrm{~g} / \mathrm{dL}$ ), female gender, the number of previous CIED-related procedures and age below 30 years at first CIED implantation. The numbers of expected major complications in the two groups were determined using the SAFeTY TLE score calculator, an online tool available at http://alamay2.linuxpl.info/kalkulator/ [15].

\subsection{Statistical Analysis}

The Shapiro-Wilk test showed that most continuous variables were normally distributed. For uniformity, all continuous variables are presented as the mean \pm standard deviation. The categorical variables are presented as a number and percentage. The significance of differences between groups was determined using the nonparametric $\mathrm{chi}^{2}$ test with Yates correction or the unpaired " $\mathrm{U}$ " Mann-Whitney test, as appropriate.

The Kaplan-Meier method was used to calculate the probability of mortality-free survival in patients divided into two groups according to the presence or absence of TEE monitoring during TLE. The log-rank test, including complete and censored data, was used to test for differences between the survival curves. A two-tailed $p$ value $<0.05$ was considered statistically significant. Statistical analysis was performed with Statistica version 13.3 (TIBCO Software Inc., AP Palo Alto, CA, USA).

\section{Results}

\subsection{Clinical Characteristics of the Study Population}

TEE-guided patients were older and more frequently had comorbidities (Charlson comorbidity index $4.972 \pm 3.756$ vs. $4.434 \pm 3.538 ; p<0.001$ ) compared with non-TEE-guided individuals. Those in the TEE-guided group more often had lower left ventricular ejection fraction (LVEF) and higher New York Heart Assiocation (NYHA) functional class. Moreover, TEE-guided patients were characterized by lower blood levels of hemoglobin and were more frequently treated for chronic renal failure. The groups did not differ significantly in the incidence of other diseases such as diabetes mellitus, coronary artery disease, previous sternotomy or chronic atrial fibrillation, whereas arterial hypertension was more common in non-TEE-guided individuals (60.779 vs. 53.012; $p<0.001)$. Patient age at first CIED implantation was comparable between the groups: $58.096 \pm 15.813$ vs. $57.348 \pm 17.628 ; p=0.558$, whereas patients in the TEE-guided group were older at the time of lead extraction (67.419 $\pm 14.232 \mathrm{vs}$. $64.849 \pm 16.223 ; p<0.001$ ) (Table 1). 
Table 1. Demographic and clinical information.

\begin{tabular}{|c|c|c|c|c|}
\hline Parameter & All Group & $\begin{array}{l}\text { With TEE } \\
\text { Monitoring }\end{array}$ & $\begin{array}{l}\text { Without TEE } \\
\text { Monitoring }\end{array}$ & $\begin{array}{c}\text { Mann-Whitney } \\
\text { “U” Test/chi }{ }^{2} \text { Test }\end{array}$ \\
\hline Number of patients & 3185 & 1079 & 2106 & \\
\hline $\begin{array}{l}\text { Patient age during TLE (years); } \\
\qquad x \pm S D\end{array}$ & $\begin{array}{c}65.720 \\
\pm 15.622 \\
N=3185\end{array}$ & $\begin{array}{c}67.419 \\
\pm 14.232 \\
N=1079\end{array}$ & $\begin{array}{c}64.849 \\
\pm 16.223 \\
\mathrm{~N}=2106\end{array}$ & $p<0.001$ \\
\hline $\begin{array}{l}\text { Patient age at first implantation } \\
\text { (years); } \mathrm{x} \pm \mathrm{SD}\end{array}$ & $\begin{array}{c}57.601 \\
\pm 17.036 \\
N=3185\end{array}$ & $\begin{array}{c}58.096 \\
\pm 15.813 \\
\mathrm{~N}=1079\end{array}$ & $\begin{array}{c}57.348 \\
\pm 17.628 \\
N=2106\end{array}$ & $p=0.558$ \\
\hline NYHA class; $x \pm$ SD & $\begin{array}{c}1.797 \\
\pm 0.692 \\
\mathrm{~N}=3185\end{array}$ & $\begin{array}{c}2.035 \\
\pm 0.564 \\
\mathrm{~N}=1079\end{array}$ & $\begin{array}{c}1.676 \\
\pm 0.719 \\
\mathrm{~N}=2106\end{array}$ & $p<0.001$ \\
\hline NYHA class III \& IV; n (\%) & $\begin{array}{c}451 \\
(14.160) \\
\mathrm{N}=3185\end{array}$ & $\begin{array}{c}173 \\
(16.033) \\
\mathrm{N}=1079\end{array}$ & $\begin{array}{c}278 \\
(13.200) \\
\mathrm{N}=2106\end{array}$ & $p<0.05$ \\
\hline LVEF $[\%] ; x \pm S D$ & $\begin{array}{c}48.911 \\
\pm 15.053 \\
N=3145\end{array}$ & $\begin{array}{c}48.795 \\
\pm 15.852 \\
N=1070\end{array}$ & $\begin{array}{c}48.971 \\
\pm 14.628 \\
\mathrm{~N}=2075\end{array}$ & $p=0.896$ \\
\hline $\mathrm{LVEF}<40 \%$; n (\%) & $\begin{array}{c}879 \\
(27.598) \\
N=3185\end{array}$ & $\begin{array}{c}331 \\
(30.677) \\
N=1079\end{array}$ & $\begin{array}{c}548 \\
(26.021) \\
N=2106\end{array}$ & $p<0.01$ \\
\hline $\begin{array}{l}\text { Hemoglobin concentration; } \\
\qquad(\mathrm{g} / \mathrm{dL} 0 \mathrm{x} \pm \mathrm{SD}\end{array}$ & $\begin{array}{c}12.901 \\
\pm 1.946 \\
\mathrm{~N}=3121\end{array}$ & $\begin{array}{c}12.589 \\
\pm 2.007 \\
\mathrm{~N}=1059\end{array}$ & $\begin{array}{c}13.062 \\
\pm 1.894 \\
\mathrm{~N}=2062 \\
\end{array}$ & $p<0.001$ \\
\hline $\begin{array}{l}\text { Renal failure, creatinine } \\
\text { concentration }>1.3 \mathrm{mg} / \mathrm{dL} ; \mathrm{n}(\%)\end{array}$ & $\begin{array}{c}680 \\
(21.601) \\
N=3148\end{array}$ & $\begin{array}{c}262 \\
(24.764) \\
\mathrm{N}=1058\end{array}$ & $\begin{array}{c}418 \\
(20.000) \\
N=2090\end{array}$ & $p<0.01$ \\
\hline Diabetes mellitus; n (\%) & $\begin{array}{c}624 \\
(19.592) \\
N=3185\end{array}$ & $\begin{array}{c}229 \\
(21.223) \\
\mathrm{N}=1079\end{array}$ & $\begin{array}{c}395 \\
(18.756) \\
\mathrm{N}=2106\end{array}$ & $p=0.107$ \\
\hline Arterial hypertension; $\mathrm{n}(\%)$ & $\begin{array}{c}1852 \\
(58.148) \\
N=3185\end{array}$ & $\begin{array}{c}572 \\
(53.012) \\
\mathrm{N}=1079\end{array}$ & $\begin{array}{c}1280 \\
(60.779) \\
N=2106\end{array}$ & $p<0.001$ \\
\hline $\begin{array}{c}\text { Coronary artery } \\
\text { disease/stroke/peripheral artery } \\
\text { disease } \mathrm{n} ; \mathrm{n}(\%)\end{array}$ & $\begin{array}{c}1328 \\
(41.695) \\
N=3185\end{array}$ & $\begin{array}{c}455 \\
(42.169) \\
\mathrm{N}=1079\end{array}$ & $\begin{array}{c}873 \\
(41.453) \\
\mathrm{N}=2106\end{array}$ & $p=0.720$ \\
\hline Permanent atrial fibrillation; n (\%) & $\begin{array}{c}724 \\
(22.732) \\
\mathrm{N}=3185 \\
\end{array}$ & $\begin{array}{c}257 \\
(23.818) \\
N=1079 \\
\end{array}$ & $\begin{array}{c}467 \\
(22.175) \\
N=2106\end{array}$ & $p=0.316$ \\
\hline Prior sternotomy; n (\%) & $\begin{array}{c}478 \\
(15.008) \\
N=3185\end{array}$ & $\begin{array}{c}145 \\
(13.438) \\
\mathrm{N}=1079\end{array}$ & $\begin{array}{c}333 \\
(15.812) \\
N=2106\end{array}$ & $p=0.085$ \\
\hline Charlson comorbidity index; $x \pm S D$ & $\begin{array}{c}4.616 \\
\pm 3.621 \\
\mathrm{~N}=3178\end{array}$ & $\begin{array}{c}4.972 \\
\pm 3.756 \\
\mathrm{~N}=1072\end{array}$ & $\begin{array}{c}4.434 \\
\pm 3.538 \\
\mathrm{~N}=2106\end{array}$ & $p<0.001$ \\
\hline
\end{tabular}

$\overline{\text { Abbreviations: left ventricular ejection fraction (LVEF), N New York Heart Association (NYHSA), transvenous lead }}$ extraction (TLE).

\subsection{Preprocedural Data on CIED}

Noninfectious indications for TLE were most frequent in the TEE-guided group (77.943\% vs. $60.351 \% ; p<0.001$ ), whereas infectious indications were more common in the non-TEE-guided group; both lead-related infective endocarditis (LRIE) and pocket infection were more frequent in patients without intraprocedural TEE $(27.635 \%$ vs. $15.477 \%$; $p<0.001$ and $12.013 \%$ vs. $6.580 \% ; p<0.001$, respectively). The number of implanted leads was similar in both groups. TEE-guided patients had older leads: the lead dwell time of the oldest leads before TLE was $112.665 \pm 75.955$ vs. $90.807 \pm 69.959$ months; $p<0.001$. Furthermore, patients undergoing TLE under echocardiographic guidance had more ICDs or CSs $(35.125 \%$ vs. $30.009 \% ; p<0.01)$, and fewer abandoned leads $(0.087 \pm 0.282$ vs. $0.135 \pm 0.342 ; p<0.05$ ) than those without intraprocedural TEE (Table 2). 
Table 2. Indications for TLE and preoperative data on cardiac implantable electronic devices (CIED).

\begin{tabular}{|c|c|c|c|c|}
\hline Parameter & All Group & $\begin{array}{l}\text { With TEE } \\
\text { Monitoring }\end{array}$ & $\begin{array}{l}\text { Without TEE } \\
\text { Monitoring }\end{array}$ & $\begin{array}{l}\text { Mann-Whitney } \\
\text { "U" Test/chi }{ }^{2} \text { Test }\end{array}$ \\
\hline Number of patients & 3185 & 1079 & 2106 & \\
\hline \multicolumn{5}{|c|}{ Indications for TLE } \\
\hline $\begin{array}{l}\text { LRIE certain or probable with or without } \\
\text { pocket infection; } \mathrm{n}(\%)\end{array}$ & $\begin{array}{c}749 \\
(23.516) \\
N=3185\end{array}$ & $\begin{array}{c}167 \\
(15.477) \\
N=1079\end{array}$ & $\begin{array}{c}582 \\
(27.635) \\
N=2106\end{array}$ & $p<0.001$ \\
\hline Local (pocket) infection (only); n (\%) & $\begin{array}{c}324 \\
(10.173) \\
\mathrm{N}=3185\end{array}$ & $\begin{array}{c}71 \\
(6.580) \\
N=1079\end{array}$ & $\begin{array}{c}253 \\
(12.013) \\
\mathrm{N}=2106\end{array}$ & $p<0.001$ \\
\hline Non-infectious indications, all; n (\%) & $\begin{array}{c}2112 \\
(66.311) \\
\mathrm{N}=3185\end{array}$ & $\begin{array}{c}841 \\
(77.943) \\
\mathrm{N}=1079\end{array}$ & $\begin{array}{c}1271 \\
(60.351) \\
\mathrm{N}=2106\end{array}$ & $p<0.001$ \\
\hline $\begin{array}{l}{ }^{*} \text { Mechanical lead damage (electric } \\
\text { failure) } \mathrm{n}(\%)\end{array}$ & $\begin{array}{c}816 \\
(38.636) \\
N=2112\end{array}$ & $\begin{array}{c}337 \\
(40.071) \\
N=841\end{array}$ & $\begin{array}{c}479 \\
(37.687) \\
N=1271\end{array}$ & $p=0.291$ \\
\hline $\begin{array}{l}\text { * Lead dysfunction (exit/entry block, } \\
\text { dislodgement, extracardiac pacing) n (\%) }\end{array}$ & $\begin{array}{c}387 \\
(18.324) \\
\mathrm{N}=2112\end{array}$ & $\begin{array}{c}159 \\
(18.906) \\
\mathrm{N}=841\end{array}$ & $\begin{array}{c}228 \\
(17.939) \\
\mathrm{N}=1271\end{array}$ & $p=0.614$ \\
\hline $\begin{array}{l}{ }^{*} \text { Lead dysfunction caused by (usually } \\
\text { dry)—perforation } n(\%)\end{array}$ & $\begin{array}{c}350 \\
(16.572) \\
N=2112\end{array}$ & $\begin{array}{c}143 \\
(17.004) \\
\mathrm{N}=841\end{array}$ & $\begin{array}{c}207 \\
(16.286) \\
N=1271\end{array}$ & $p=0.708$ \\
\hline $\begin{array}{c}\text { * Change of pacing mode/upgrading, } \\
\text { downgrading } n(\%)\end{array}$ & $\begin{array}{c}168 \\
(7.955) \\
N=2112\end{array}$ & $\begin{array}{c}66 \\
(7.848) \\
\mathrm{N}=841\end{array}$ & $\begin{array}{c}102 \\
(8.025) \\
N=1271\end{array}$ & $p=0.948$ \\
\hline $\begin{array}{c}* \text { Abandoned lead/prevention of } \\
\text { abandonment (AF, overmuch of leads) } n \\
(\%)\end{array}$ & $\begin{array}{c}99 \\
(4.688) \\
\mathrm{N}=2112\end{array}$ & $\begin{array}{c}28 \\
(3.329) \\
\mathrm{N}=841\end{array}$ & $\begin{array}{c}71 \\
(5.586) \\
N=1271\end{array}$ & $p<0.05$ \\
\hline $\begin{array}{l}\text { * Threatened/potentially threatened lead } \\
\text { (loops, free ending, left heart, LDTD) } \mathrm{n} \\
(\%)\end{array}$ & $\begin{array}{c}89 \\
(4.214)\end{array}$ & $\begin{array}{c}36 \\
(4.281) \\
\mathrm{N}=841\end{array}$ & $\begin{array}{c}53 \\
(4.170) \\
\mathrm{N}=1271\end{array}$ & $p=0.989$ \\
\hline $\begin{array}{c}\text { * Other (MRI indication, cancer, pain of } \\
\text { pocket, loss of indication for pacing / ICD) } \\
n(\%)\end{array}$ & $\begin{array}{c}72 \\
(3.409) \\
\mathrm{N}=2112\end{array}$ & $\begin{array}{c}31 \\
(3.686) \\
\mathrm{N}=841\end{array}$ & $\begin{array}{c}41 \\
(3.226) \\
\mathrm{N}=1271\end{array}$ & $p=0.654$ \\
\hline $\begin{array}{l}\text { * Recapture venous access (symptomatic. } \\
\text { occlusion, SVC syndrome, lead } \\
\text { replacement/upgrading) n (\%) }\end{array}$ & $\begin{array}{c}131 \\
(6.203) \\
N=2112\end{array}$ & $\begin{array}{c}41 \\
(4.875) \\
\mathrm{N}=841\end{array}$ & $\begin{array}{c}90 \\
(7.081) \\
\mathrm{N}=1271\end{array}$ & $p<0.05$ \\
\hline \multicolumn{5}{|c|}{ Preoperative data on CIED } \\
\hline $\begin{array}{l}\text { Dwell time of oldest lead in patients } \\
\text { before TLE (months); } x \pm S D\end{array}$ & $\begin{array}{c}98.212 \\
\pm 72.773 \\
\mathrm{~N}=3185\end{array}$ & $\begin{array}{c}112.665 \\
\pm 75.955 \\
\mathrm{~N}=1079\end{array}$ & $\begin{array}{c}90.807 \\
\pm 69.959 \\
N=2106\end{array}$ & $p<0.001$ \\
\hline $\begin{array}{l}\text { Mean implant duration before TLE } \\
\text { (months); } \pm \text { SD }\end{array}$ & $\begin{array}{c}90.050 \\
\pm 63.656 \\
\mathrm{~N}=3185\end{array}$ & $\begin{array}{c}105.235 \\
\pm 67.991 \\
\mathrm{~N}=1079\end{array}$ & $\begin{array}{c}82.271 \\
\pm 59.858 \\
N=2106\end{array}$ & $p<0.001$ \\
\hline CIED with ICD or CS lead; n (\%) & $\begin{array}{c}1011 \\
(31.743) \\
\mathrm{N}=3185\end{array}$ & $\begin{array}{c}379 \\
(35.125) \\
\mathrm{N}=1079\end{array}$ & $\begin{array}{c}632 \\
(30.009) \\
N=2106\end{array}$ & $p<0.01$ \\
\hline $\begin{array}{l}\text { Number of abandoned leads before TLE; } \\
\qquad x \pm S D\end{array}$ & $\begin{array}{c}0.119 \\
\pm 0.323 \\
\mathrm{~N}=3185\end{array}$ & $\begin{array}{c}0.087 \\
\pm 0.282 \\
\mathrm{~N}=1079\end{array}$ & $\begin{array}{c}0.135 \\
\pm 0.342 \\
\mathrm{~N}=2106\end{array}$ & $p<0.05$ \\
\hline $\begin{array}{l}\text { Presence of abandoned lead(s) before } \\
\text { TLE; } \mathrm{n}(\%)\end{array}$ & $\begin{array}{c}379 \\
(11.900) \\
\mathrm{N}=3185\end{array}$ & $\begin{array}{c}95 \\
(8.804) \\
\mathrm{N}=1079\end{array}$ & $\begin{array}{c}284 \\
(13.485) \\
N=2106\end{array}$ & $p<0.001$ \\
\hline Abandoned leads only (EPM. EHV); n (\%) & $\begin{array}{c}32 \\
(1.004) \\
\mathrm{N}=3185\end{array}$ & $\begin{array}{c}4 \\
(0.371) \\
\mathrm{N}=1079\end{array}$ & $\begin{array}{c}28 \\
(1.330) \\
\mathrm{N}=2106\end{array}$ & $p<0.05$ \\
\hline $\begin{array}{l}\text { Number of leads in the system before } \\
\text { TLE; } x \pm S D\end{array}$ & $\begin{array}{c}1.817 \\
\pm 0.634 \\
\mathrm{~N}=3161\end{array}$ & $\begin{array}{c}1.829 \\
\pm 0.622 \\
\mathrm{~N}=1076\end{array}$ & $\begin{array}{c}1.812 \\
\pm 0.640 \\
\mathrm{~N}=2085\end{array}$ & $p=0.400$ \\
\hline $\begin{array}{l}\text { Number of leads in the heart before TLE; } \\
\qquad x \pm S D\end{array}$ & $\begin{array}{c}1.962 \\
\pm 0.758 \\
\mathrm{~N}=3185\end{array}$ & $\begin{array}{c}1.930 \\
\pm 0.715 \\
\mathrm{~N}=1079\end{array}$ & $\begin{array}{c}1.978 \\
\pm 0.779 \\
\mathrm{~N}=2106\end{array}$ & $p=0.324$ \\
\hline
\end{tabular}

* Main non-infectious indication. Abbreviations: atrial fibrillation (AF), cardiac implantable electronic device (CIED), coronary sinus (CS), defibrillator lead without previously removed unit (EHV), pacemaker lead without previously removed unit (EPM), implantable cardioverter-defibrillator (ICD), lead-related infective endocarditis (LRIE), lead-dependent tricuspid dysfunction (LDTD), magnetic resonance imaging (MRI). 


\subsection{Outcomes of Transvenous Lead Extraction}

A total of 5258 leads were extracted, including 1760 in the TEE-guided group and 3498 in the non-TEE-guided group. In both groups, the number of extracted leads per patient was similar on average, $1.631 \pm 0.719$ vs. $1.661 \pm 0.761 ; p=0.477$. The TEE-guided group differed from the non-TEE-guided group by the older age of the extracted leads (sum of lead dwell times: $15.466 \pm 13.960$ vs. $11.876 \pm 11.004$ years; $p<0.001)$, more frequent removal of ICD leads $(31.603 \%$ vs. $25.309 \%$; $p<0.001)$, less frequent extraction of left ventricular leads $(11.307 \%$ vs. $14.008, p<0.05)$ and abandoned leads $(8.804 \%$ vs. $15.527 \% ; p<0.001)$. Technical difficulties during the procedures and additional tools for lead removal were more common in the TEE-guided group $(23.818 \%$ vs. $15.907 \% ; p<0.001$ and $6.951 \%$ vs. $5.128 \% ; p<0.05$, respectively), whereas modification of venous access was more frequently necessary in non-TEE-guided individuals (5.558 vs. $1.668 ; p<0.001)$. The duration of the procedure, measured by various parameters (skin-to-skin, sheath-to-sheath and mean duration of one lead extraction), was significantly longer in the TEE-guided group.

Complete procedural success was significantly higher in the TEE-guided group (97.683\% vs. $95.442 \%, p<0.01$ ), whereas clinical success was comparable between the groups. In the periprocedural period, there was not a single death among TEE-guided patients, compared with six deaths $(0.285 \%)$ in the non-TEE-guided group ( $p=0.186$ ) (Table 3$)$.

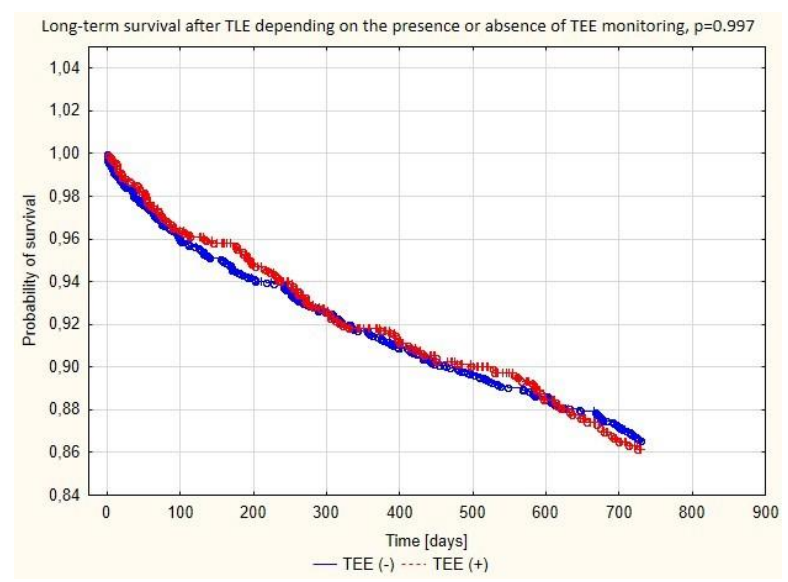

Figure 1. The Kaplan-Meier curve showing the probability of survival after transvenous lead extraction (TLE) in patients with and without transesophageal echocardiography (TEE) monitoring.

Table 3. Data on TLE procedure.

\begin{tabular}{|c|c|c|c|c|}
\hline Parameter & All Group & $\begin{array}{l}\text { With TEE } \\
\text { Monitoring }\end{array}$ & $\begin{array}{l}\text { Without TEE } \\
\text { Monitoring }\end{array}$ & $\begin{array}{l}\text { Mann-Whitney } \\
\text { "U" Test/chi' }{ }^{2} \text { Test }\end{array}$ \\
\hline Number of patients & 3185 & 1079 & 2106 & \\
\hline Number of extracted leads & 5258 & 1760 & 3498 & \\
\hline Oldest extracted lead (years); $x \pm S D$ & $\begin{array}{c}8.021 \\
\pm 5.962 \\
\mathrm{~N}=3185\end{array}$ & $\begin{array}{c}9.290 \\
\pm 6.307 \\
\mathrm{~N}=1079\end{array}$ & $\begin{array}{c}7.372 \\
\pm 5.670 \\
N=2106\end{array}$ & $p<0.001$ \\
\hline $\begin{array}{l}\text { Sum of dwell times of extracted leads } \\
\text { (years); } \pm \text { SD }\end{array}$ & $\begin{array}{c}13.091 \\
\pm 12.202 \\
\mathrm{~N}=3185\end{array}$ & $\begin{array}{c}15.466 \\
\pm 13.960 \\
N=1079\end{array}$ & $\begin{array}{c}11.876 \\
\pm 11.004 \\
\mathrm{~N}=2106\end{array}$ & $p<0.001$ \\
\hline $\begin{array}{l}\text { Number of extracted leads in one patient; } \\
\qquad x \pm S D\end{array}$ & $\begin{array}{c}1.651 \\
\pm 0.747 \\
\mathrm{~N}=3185\end{array}$ & $\begin{array}{c}1.631 \\
\pm 0.719 \\
\mathrm{~N}=1079\end{array}$ & $\begin{array}{c}1.661 \\
\pm 0.761 \\
N=2106\end{array}$ & $p=0.477$ \\
\hline HV therapy (ICD) lead was extracted; n (\%) & $\begin{array}{c}874 \\
(27.441) \\
\mathrm{N}=3185\end{array}$ & $\begin{array}{c}341 \\
(31.603) \\
\mathrm{N}=1079\end{array}$ & $\begin{array}{c}533 \\
(25.309) \\
\mathrm{N}=2106\end{array}$ & $p<0.001$ \\
\hline CS (LV pacing) lead was extracted; n (\%) & $\begin{array}{c}417 \\
(13.093) \\
\mathrm{N}=3185\end{array}$ & $\begin{array}{c}122 \\
(11.307) \\
N=1079\end{array}$ & $\begin{array}{c}295 \\
(14.008) \\
N=2106\end{array}$ & $p<0.05$ \\
\hline
\end{tabular}


Table 3. Cont.

\begin{tabular}{|c|c|c|c|c|}
\hline Parameter & All Group & $\begin{array}{l}\text { With TEE } \\
\text { Monitoring }\end{array}$ & $\begin{array}{l}\text { Without TEE } \\
\text { Monitoring }\end{array}$ & $\begin{array}{l}\text { Mann-Whitney } \\
\text { "U" Test/chi' }{ }^{2} \text { Test }\end{array}$ \\
\hline Extraction of abandoned lead(s) (any); n (\%) & $\begin{array}{c}422 \\
(13.250) \\
\mathrm{N}=3185\end{array}$ & $\begin{array}{c}95 \\
(8.804) \\
N=1079\end{array}$ & $\begin{array}{c}327 \\
(15.527) \\
\mathrm{N}=2106\end{array}$ & $p<0.001$ \\
\hline Lead fracture during extraction; $\mathrm{n}(\%)$ & $\begin{array}{c}137 \\
(4.301) \\
\mathrm{N}=3185\end{array}$ & $\begin{array}{c}51 \\
(4.727) \\
\mathrm{N}=1079\end{array}$ & $\begin{array}{c}86 \\
(4.084) \\
N=2106\end{array}$ & $p=0.451$ \\
\hline Loss of free lead fragment; $n(\%)$ & $\begin{array}{c}15 \\
(0.471) \\
\mathrm{N}=3185\end{array}$ & $\begin{array}{c}7 \\
(0.648) \\
\mathrm{N}=1079\end{array}$ & $\begin{array}{c}8 \\
(0.380) \\
N=2106\end{array}$ & $p=0.438$ \\
\hline Technical difficulty during TLE (any); n (\%) & $\begin{array}{c}592 \\
(18.587) \\
\mathrm{N}=3185\end{array}$ & $\begin{array}{c}257 \\
(23.818) \\
\mathrm{N}=1079\end{array}$ & $\begin{array}{c}335 \\
(15.907) \\
N=2106\end{array}$ & $p<0.001$ \\
\hline Two or more technical difficulties; $\mathrm{n}(\%)$ & $\begin{array}{c}127 \\
(3.987) \\
\mathrm{N}=3185\end{array}$ & $\begin{array}{c}75 \\
(6.951) \\
\mathrm{N}=1079\end{array}$ & $\begin{array}{c}52 \\
(2.469) \\
\mathrm{N}=2106\end{array}$ & $p<0.001$ \\
\hline Necessity to change venous approach; n (\%) & $\begin{array}{c}135 \\
(4.240) \\
\mathrm{N}=3184\end{array}$ & $\begin{array}{c}18 \\
(1.668) \\
N=1079\end{array}$ & $\begin{array}{c}117 \\
(5.558) \\
\mathrm{N}=2105\end{array}$ & $p<0.001$ \\
\hline $\begin{array}{l}\text { Use of other than extracted lead venous } \\
\text { approach; } n(\%)\end{array}$ & $\begin{array}{c}173 \\
(5.432) \\
\mathrm{N}=3185\end{array}$ & $\begin{array}{c}28 \\
(2.595) \\
\mathrm{N}=1079\end{array}$ & $\begin{array}{c}145 \\
(6.885) \\
N=2106\end{array}$ & $p<0.001$ \\
\hline $\begin{array}{l}\text { Necessity to use other than Byrd dilator } \\
\text { tools (Evo, TightR, lassos, basket catheters); } \\
\text { n (\%) }\end{array}$ & $\begin{array}{c}183 \\
(5.746) \\
\mathrm{N}=3185\end{array}$ & $\begin{array}{c}75 \\
(6.951) \\
\mathrm{N}=1079\end{array}$ & $\begin{array}{c}108 \\
(5.128) \\
\mathrm{N}=2106\end{array}$ & $p<0.05$ \\
\hline $\begin{array}{l}\text { Procedure duration (skin-to-skin); minutes } \\
\qquad x \pm S D\end{array}$ & $\begin{array}{c}59.952 \\
\pm 25.717 \\
\mathrm{~N}=3185\end{array}$ & $\begin{array}{c}62.747 \\
\pm 27.138 \\
\mathrm{~N}=1079\end{array}$ & $\begin{array}{c}58.520 \\
\pm 24.842 \\
\mathrm{~N}=2106\end{array}$ & $p<0.001$ \\
\hline $\begin{array}{l}\text { Procedure duration (sheath-to-sheath time); } \\
\qquad \begin{array}{l}\text { minutes } \\
\mathrm{x} \pm \mathrm{SD}\end{array}\end{array}$ & $\begin{array}{c}15.125 \\
\pm 23.274 \\
\mathrm{~N}=3185\end{array}$ & $\begin{array}{c}15.374 \\
\pm 24.396 \\
\mathrm{~N}=1079\end{array}$ & $\begin{array}{c}14.997 \\
\pm 22.682 \\
\mathrm{~N}=2106\end{array}$ & $p<0.001$ \\
\hline $\begin{array}{l}\text { Procedure duration average single lead } \\
\quad \text { extraction time; minutes } x \pm S D\end{array}$ & $\begin{array}{c}8.965 \\
\pm 12.418 \\
\mathrm{~N}=3182\end{array}$ & $\begin{array}{c}9.111 \\
\pm 14.260 \\
\mathrm{~N}=1079\end{array}$ & $\begin{array}{c}8.891 \\
\pm 11.363 \\
\mathrm{~N}=2103\end{array}$ & $p<0.01$ \\
\hline $\begin{array}{l}\text { Partial radiological success (retained tip or } \\
\quad<4 \mathrm{~cm} \text { lead fragment); } \mathrm{n}(\%)\end{array}$ & $\begin{array}{c}136 \\
(4.270) \\
\mathrm{N}=3185\end{array}$ & $\begin{array}{c}34 \\
(3.151) \\
\mathrm{N}=1079\end{array}$ & $\begin{array}{c}102 \\
(4.843) \\
N=2106\end{array}$ & $p<0.05$ \\
\hline Complete clinical success; n (\%) & $\begin{array}{c}3116 \\
(97.834) \\
\mathrm{N}=3185\end{array}$ & $\begin{array}{c}1056 \\
(97.868) \\
N=1079\end{array}$ & $\begin{array}{c}2060 \\
(97.816) \\
N=2106\end{array}$ & $p=0.975$ \\
\hline Complete procedural success; $\mathrm{n}(\%)$ & $\begin{array}{c}3064 \\
(96.201) \\
N=3185\end{array}$ & $\begin{array}{c}1054 \\
(97.683) \\
N=1079\end{array}$ & $\begin{array}{c}2010 \\
(95.442) \\
N=2106\end{array}$ & $p<0.01$ \\
\hline $\begin{array}{l}\text { Procedure-related death (intra-, } \\
\text { post-procedural); } \mathrm{n}(\%)\end{array}$ & $\begin{array}{c}6 \\
(0.188) \\
\mathrm{N}=3185\end{array}$ & $\begin{array}{c}0 \\
(0.000) \\
\mathrm{N}=1079\end{array}$ & $\begin{array}{c}6 \\
(0.285) \\
N=2106\end{array}$ & $p=0.186$ \\
\hline Died within 6 months; n (\%) & $\begin{array}{c}166 \\
5.212 \\
\mathrm{~N}=3185\end{array}$ & $\begin{array}{c}48 \\
(4.449) \\
\mathrm{N}=1079\end{array}$ & $\begin{array}{c}118 \\
(5.603) \\
N=2106\end{array}$ & $p=0.193$ \\
\hline Died within one year; n (\%) & $\begin{array}{c}263 \\
(8.257) \\
\mathrm{N}=3185\end{array}$ & $\begin{array}{c}83 \\
(7.692) \\
\mathrm{N}=1079\end{array}$ & $\begin{array}{c}180 \\
(8.547) \\
\mathrm{N}=2106\end{array}$ & $p=0.446$ \\
\hline
\end{tabular}

Abbreviations: coronary sinus (CV), left ventricle (LV), high voltage (HV), transvenous lead extraction (TLE). Mortality at 6 and 12 months after TLE was similar in both groups (Figure 1).

\subsection{Assessment of the Risk of Major Complications Using the SAFeTY TLE Score}

The total score on the SAFeTY TLE scale was higher in the TEE-guided group ( $6.143 \pm 4.395 \mathrm{vs}$. $5.593 \pm 4.127 ; p=0.004)$. The higher risk of major complications in this group was associated with longer lead dwell times and lower levels of hemoglobin $(p<0.001)$. According to the simulation, the risk of cardiac and venous perforation was significantly higher in individuals undergoing intraprocedural TEE monitoring: 1.898 (95\% CI: 1.664-2.132) vs. 1.630 (95\% CI: $1.472-1.788) ; p=0.002$. The SAFeTY 
TLE score yielded an expected number of 21 and 34 perforations in the superior vena cava (SVC), right atrium (RA) and right ventricle (RV) during TLE in the TEE-guided and non-TEE-guided group, respectively (Table 4).

Table 4. Simulation of major complications according to the SAFeTY TLE score in the validation cohort.

\begin{tabular}{|c|c|c|c|c|c|}
\hline $\begin{array}{l}\text { Simulation of Major Complications } \\
\text { According to SAFeTY TLE Score }\end{array}$ & & All Group & $\begin{array}{l}\text { With TEE } \\
\text { Monitoring }\end{array}$ & $\begin{array}{l}\text { Without TEE } \\
\text { Monitoring }\end{array}$ & $\begin{array}{l}\text { Mann-Whitney } \\
\text { "U" Test/chi' }{ }^{2} \text { Test }\end{array}$ \\
\hline Number of patients & & 3185 & 1079 & 2106 & \\
\hline $\begin{array}{l}\text { Hemoglobin concentration } \leq 11.5 \mathrm{~g} / \mathrm{dL} ; \\
\mathrm{n}(\%)\end{array}$ & A & $\begin{array}{c}710 \\
(22.749) \\
\mathrm{N}=3121\end{array}$ & $\begin{array}{c}299 \\
(27.711) \\
\mathrm{N}=1059\end{array}$ & $\begin{array}{c}411 \\
(19.932) \\
\mathrm{N}=2062\end{array}$ & $p<0.001$ \\
\hline Female; n (\%) & $\mathrm{Fe}$ & $\begin{array}{c}1231 \\
(38.650) \\
\mathrm{N}=3185\end{array}$ & $\begin{array}{c}410 \\
(37.998) \\
\mathrm{N}=1079\end{array}$ & $\begin{array}{c}821 \\
(38.984) \\
N=2106\end{array}$ & $p=0.616$ \\
\hline $\begin{array}{l}\text { Number of previous CIED procedures (all); } \\
\qquad x \pm S D\end{array}$ & $\mathrm{~T}$ & $\begin{array}{c}1.822 \\
\pm 1.081 \\
\mathrm{~N}=3185\end{array}$ & $\begin{array}{c}1.779 \\
\pm 0.965 \\
\mathrm{~N}=1079\end{array}$ & $\begin{array}{c}1.844 \\
\pm 1.136 \\
\mathrm{~N}=2106\end{array}$ & $p=0.852$ \\
\hline $\begin{array}{l}\text { Number of SAFeTY TLE score points; } \\
\qquad x \pm \text { SD }\end{array}$ & & $\begin{array}{c}5.777 \\
\pm 4.226 \\
\mathrm{~N}=3165\end{array}$ & $\begin{array}{c}6.143 \\
\pm 4.395 \\
\mathrm{~N}=1079\end{array}$ & $\begin{array}{c}5.593 \\
\pm 4.127 \\
\mathrm{~N}=2106\end{array}$ & 0.004 \\
\hline $\begin{array}{l}\text { Probability of perforation (SVC, RA, RV) } \\
\text { according to SAFeTY-TLE score; x (95\% CI) }\end{array}$ & & $\begin{array}{c}1.721 \\
95 \% \mathrm{CI} \\
(1.590-1.852)\end{array}$ & $\begin{array}{c}1.898 \\
95 \% \mathrm{CI} \\
(1.664-2.132)\end{array}$ & $\begin{array}{c}1.630 \\
95 \% \mathrm{CI} \\
(1.472-1.788)\end{array}$ & 0.002 \\
\hline $\begin{array}{l}\text { Calculated number of major complications } \\
\text { (perforations; SVC, RA, RV) according to } \\
\text { SAFeTY-TLE score; } \mathrm{n}(\%)\end{array}$ & & $\begin{array}{c}55 \\
(1.721) \\
\mathrm{N}=3185\end{array}$ & $\begin{array}{c}21 \\
(1.898) \\
\mathrm{N}=1079\end{array}$ & $\begin{array}{c}34 \\
(1.898) \\
\mathrm{N}=2106\end{array}$ & 0.592 \\
\hline
\end{tabular}

Abbreviations: cardiac implantable electronic device (CIED), right atrium (RA), right ventricle (RV), superior vena cava (SVC).

The incidence of major complications did not differ significantly between the TEE-guided and non-TEE-guided group: 21 cases $(1.946 \%)$ vs. 39 cases $(1.852 \%)$, including $15(1.390 \%)$ vs. $33(1.567 \%)$ cases of hemorrhagic pericardial or pleural effusion and six $(0.556 \%)$ vs. six $(0.3285 \%)$ cases of significant damage to the tricuspid valve (Table 5, Figure 2).

Table 5. Number of real major complications.

\begin{tabular}{|c|c|c|c|c|}
\hline Major Complication & All Group & $\begin{array}{l}\text { With TEE } \\
\text { Monitoring }\end{array}$ & $\begin{array}{l}\text { Without TEE } \\
\text { Monitoring }\end{array}$ & $\begin{array}{l}\text { Mann-Whitney } \\
\text { "U" Test/chi' }{ }^{2} \text { Test }\end{array}$ \\
\hline Number of patients & 3185 & 1079 & 2106 & \\
\hline $\begin{array}{l}\text { Real major complication } \\
\text { (any); } \mathrm{n}(\%)\end{array}$ & $\begin{array}{c}60 \\
(1.884) \\
\mathrm{N}=3185\end{array}$ & $\begin{array}{c}21 \\
(1.946) \\
\mathrm{N}=1079\end{array}$ & $\begin{array}{c}39 \\
(1.852) \\
\mathrm{N}=2106\end{array}$ & 0.962 \\
\hline $\begin{array}{l}\text { Real hemopericardium; } \\
\text { n (\%) }\end{array}$ & $\begin{array}{c}43 \\
(1.350) \\
\mathrm{N}=3185\end{array}$ & $\begin{array}{c}13 \\
(1.205) \\
\mathrm{N}=1079\end{array}$ & $\begin{array}{c}30 \\
(1.425) \\
\mathrm{N}=2106\end{array}$ & 0.729 \\
\hline $\begin{array}{l}\text { Real hemopericardium } \\
\text { or hemothorax; } \mathrm{n}(\%)\end{array}$ & $\begin{array}{c}48 \\
(1.507) \\
\mathrm{N}=3185\end{array}$ & $\begin{array}{c}15 \\
(1.390) \\
\mathrm{N}=1079\end{array}$ & $\begin{array}{c}33 \\
(1.567) \\
\mathrm{N}=2106\end{array}$ & 0.815 \\
\hline $\begin{array}{l}\text { Real tricuspid valve } \\
\text { damage during TLE; } \\
\mathrm{n}(\%)\end{array}$ & $\begin{array}{c}12 \\
(0.377) \\
\mathrm{N}=3185\end{array}$ & $\begin{array}{c}6 \\
(0.556) \\
N=1079\end{array}$ & $\begin{array}{c}6 \\
(0.285) \\
N=2106\end{array}$ & 0.381 \\
\hline $\begin{array}{l}\text { Rescue cardiac surgery; } \\
\text { n (\%) }\end{array}$ & $\begin{array}{c}37 \\
(1.162) \\
\mathrm{N}=3185\end{array}$ & $\begin{array}{c}16 \\
(1.483) \\
\mathrm{N}=1079\end{array}$ & $\begin{array}{c}21 \\
(0.997) \\
N=2106\end{array}$ & $p=0.300$ \\
\hline
\end{tabular}




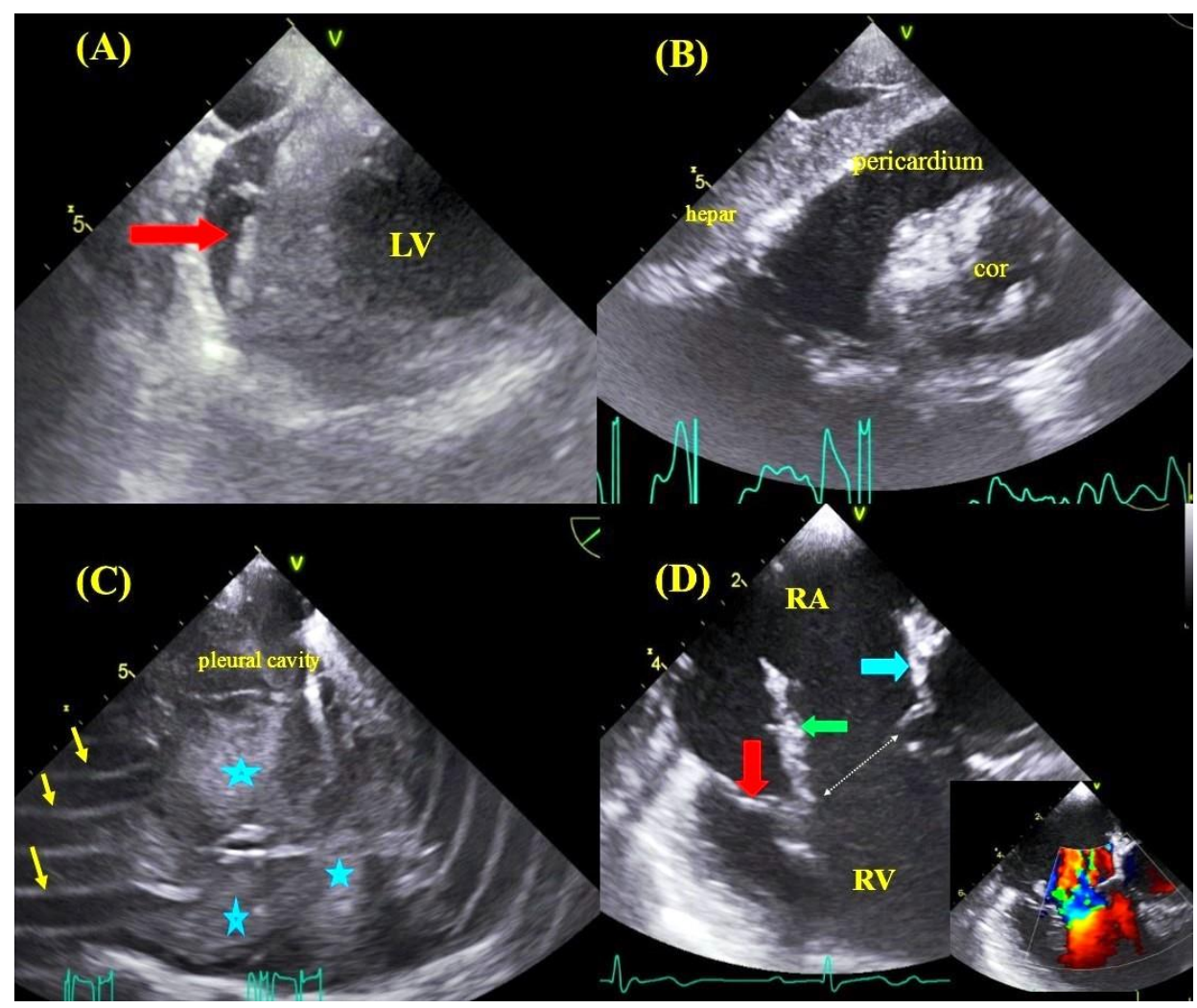

Figure 2. Possible TLE complications detected in TEE: TEE images-transgastric views, the formation of blood clot (red arrow) in the course of self-limiting bleeding into the pericardial sac (A), massive bleeding into the pericardial sac resulting in cardiac tamponade (B). TEE images-bleeding into the right pleural cavity with visible blood clots (asterisks), image blurring caused by electrocoagulation during thoracotomy (arrows) (C). TEE images-low esophageal view-injury to the tricuspid leaflets and papillary muscle rupture resulting in massive tricuspid regurgitation (color Doppler): anterior leaflet (red arrow), ruptured head of the papillary muscle (green arrow), fragment of the septal leaflet (blue arrow) (D).

Detailed analysis of major complications in both groups showed that, in TEE-guided patients, the number of major complications associated with hemorrhagic pericardial or pleural effusion was six less than predicted by the SAFeTY TLE score-a reduction of $28.57 \%(p<0.05)$. In the non-TEE-guided group there were 33 major complications vs. 34 predicted by the risk calculator (Table 6).

Table 6. Comparison of number of major complications (perforations; SVC, RA, RV), calculated vs real complications, according to data from Tables 4 and 5 .

\begin{tabular}{|c|c|c|c|c|c|}
\hline & $\begin{array}{l}\text { Number of } \\
\text { Patients }\end{array}$ & $\begin{array}{c}\text { Calculated Number of Major } \\
\text { Complications (Perforations; } \\
\text { SVC, RA, RV) According to } \\
\text { SAFeTY-TLE Score; n (\%) }\end{array}$ & $\begin{array}{c}\text { Real Number of } \\
\text { Major Complications } \\
\text { (Perforations; SVC, } \\
\text { RA, RV) n (\%) }\end{array}$ & $\begin{array}{l}\text { Change } \\
\text { N (\%) }\end{array}$ & chi $^{2}$ Test \\
\hline All group & $\mathrm{N}=3185$ & $\begin{array}{c}55 \\
(1.721) \\
\mathrm{N}=3185\end{array}$ & $\begin{array}{c}48 \\
(1.507) \\
\mathrm{N}=3185\end{array}$ & $\begin{array}{c}-7 \\
(-12.727) \\
\end{array}$ & $p<0.05$ \\
\hline $\begin{array}{l}\text { With TEE } \\
\text { monitoring }\end{array}$ & $\mathrm{N}=1079$ & $\begin{array}{c}21 \\
(1.898) \\
\mathrm{N}=1079\end{array}$ & $\begin{array}{c}15 \\
(1.390) \\
N=1079\end{array}$ & $\begin{array}{c}-6 \\
(-28.571)\end{array}$ & $p<0.05$ \\
\hline $\begin{array}{l}\text { Without TEE } \\
\text { monitoring }\end{array}$ & $N=2106$ & $\begin{array}{c}34 \\
(1.898) \\
\mathrm{N}=2106\end{array}$ & $\begin{array}{c}33 \\
(1.567) \\
\mathrm{N}=2106\end{array}$ & $\begin{array}{c}-1 \\
(2.941)\end{array}$ & $p=1.000$ \\
\hline
\end{tabular}

Abbreviations: superior vena cava (SVC), right atrium (RA) and right ventricle (RV); for the SAFeTY-TLE score, see Table 4. 


\section{Discussion}

A search of the literature revealed few small observational studies and case reports that addressed the usefulness of transesophageal echocardiography for monitoring the patient during transvenous lead extraction [16-20]. This is the first study to compare the impact of TEE guidance on the effectiveness and safety of transvenous lead extraction procedures in two large groups: 1079 patients undergoing continuous TEE monitoring and 2106 patients with echocardiographic assessment before and after the procedure. The current study found that the use of continuous echocardiographic monitoring of lead extraction procedures resulted in a significantly decreased frequency of most dangerous complications related to cardiac and vascular damage.

As is well known, age of the lead at the time of extraction is the most important risk factor for complications related to TLE. This parameter is incorporated into scoring systems for the prediction of technical difficulties during the procedure and for the stratification of the risk of major complications related to TLE $[10,15]$. Intraprocedural transesophageal echocardiography permits visualization and evaluation of structural changes caused by leads with long dwell times, thus facilitating navigation during the extraction procedure. In the initial phase of the procedure, it is very important to visualize lead adhesion to cardiac and vascular structures. TEE facilitates the imaging of lead adhesion to the walls of the superior vena cava, tricuspid valve and walls of the right atrium and right ventricle, as well as assessing lead-to-lead adhesion and the degree of fibrous tissue growth around lead tips in the cardiac wall (Figure 3).

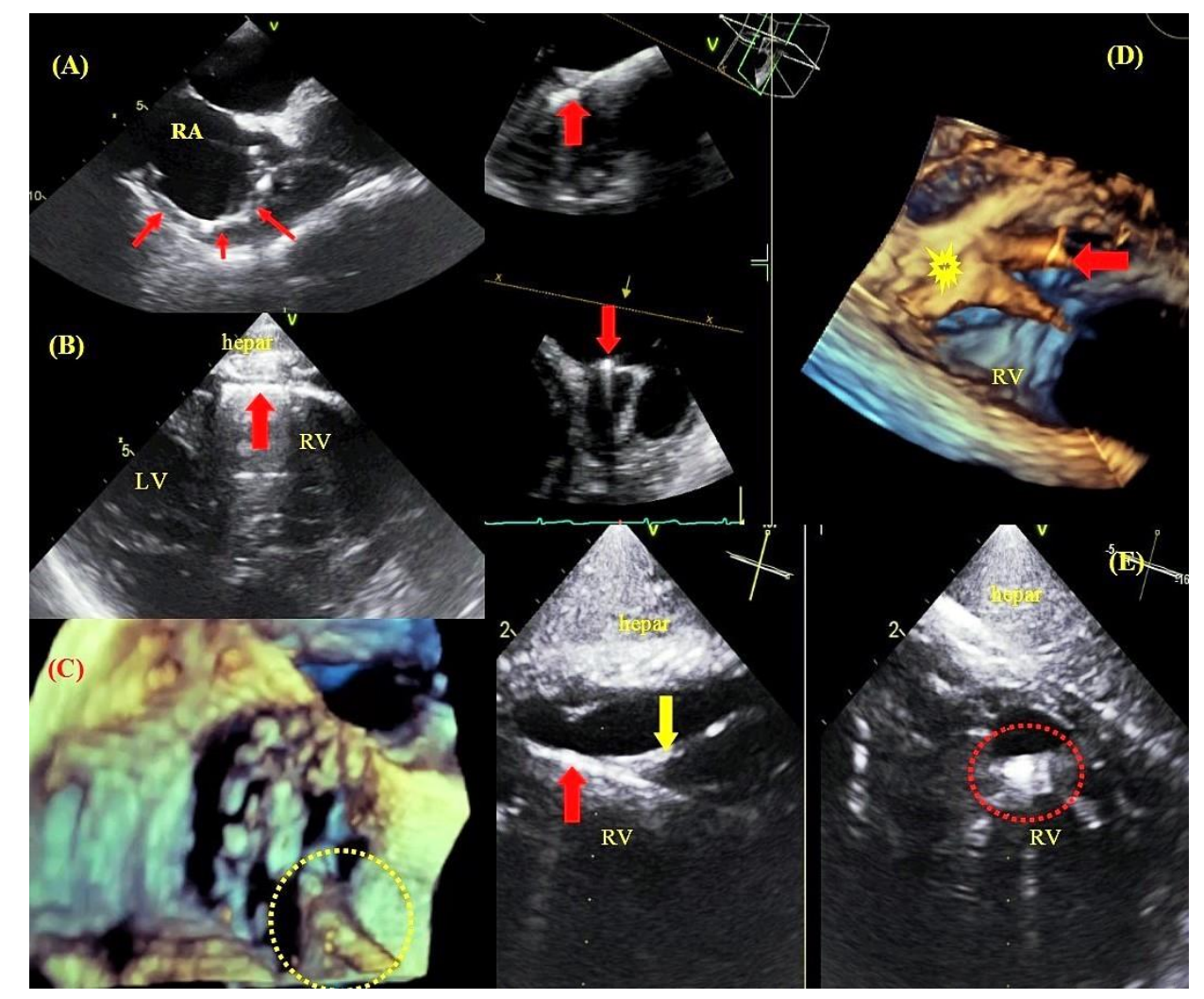

Figure 3. Binding sites between leads and cardiovascular structures visualized by TEE. TEE-bicaval view - a thickened lead (red arrows) adhered to the wall of the right atrium and the superior vena cava orifice (A). TEE - transgastric view - a ventricular lead (arrow) adhered to the right ventricular wall (B). 3D TEE imaging — the tricuspid valve with a lead adhered to the leaflet margin (C). 3D imaging (Multi-D) - a lead (red arrow) implanted at the base of the papillary muscle (yellow arrow) (D). TEE (Multi-D)—a ventricular lead (red arrow) adhered to the tendinous thread (yellow arrow) (E). 
The information provided by an echocardiographist during the procedure permits the extractor to take appropriate precautions during the freeing of the lead from the encapsulating fibrous tissue and pulling on the cardiac walls to remove the lead in its entirety.

The imaging of lead adhesions permitted us to determine the site of potential cardiac injury. An analysis of traction force and winding of soft tissues during dilator maneuvers warned the extractor of the risk of possible damage, which undoubtedly affected the procedure strategy, resulting in a reduction in the number of the most severe complications: cardiac and vascular perforations (Figures 4 and 5).

A more detailed description of the echocardiographic findings in patients with TEE monitoring during TLE was presented in an earlier report [21].

The SAFeTY TLE score of 21 for wall perforations in the TEE-guided group was significantly higher than the number of actual complications $(n=15)$, which corresponds to a reduction of $28.571 \%$; 95\% CI (9.249-47.893); $p=0.028$ in the risk of perforation. In the non-TEE-guided group, the score for perforations was 34 compared with the actual incidence of 33 complications, which confirms the accuracy of the calculation and significant benefits of using TEE as a monitoring tool during the extraction procedure. The decreased risk of damage to the superior vena cava, right atrium and right ventricle documented in patients undergoing TLE under continuous TEE guidance is certainly a result of implementing such an approach. This study supports evidence from previous small observational studies [16-20].

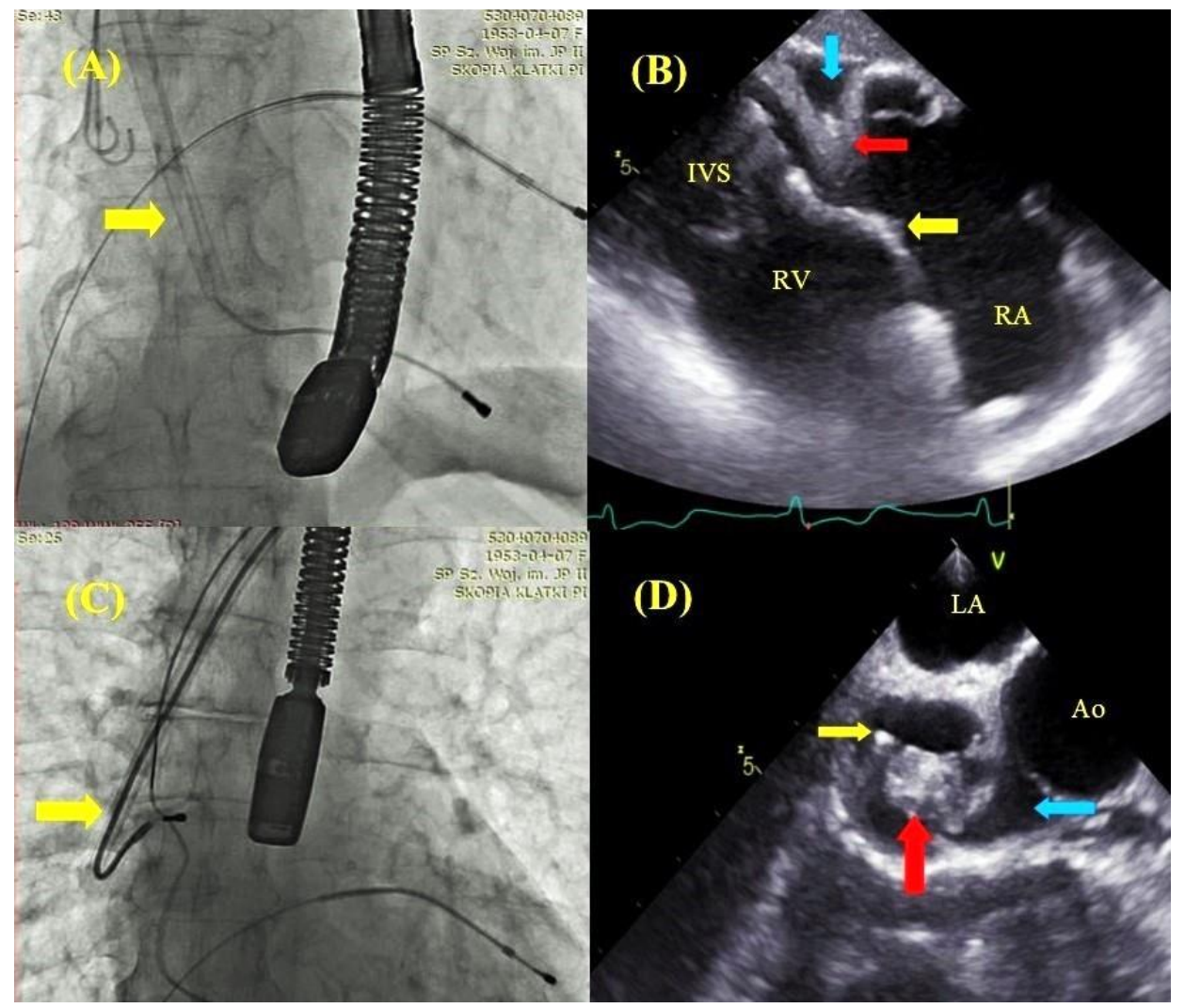

Figure 4. Comparison of fluoroscopic and echocardiographic images during lead extraction maneuvers. The moment of ventricular lead extracting; the yellow arrow points to the Byrd dilator slipped over the lead (A). TEE - transgastric view - the moment of ventricular lead extraction (A) with pulling on the right ventricular (RV) wall (red arrow), hyperechogenic thickened lead adhered to the endocardium (yellow arrow), the separation of pericardial layers corresponding to pseudo-cardiac tamponade (blue arrow) (B). The moment of atrial lead extraction; the yellow arrow points to the Byrd dilator slipped over the lead (C). TEE-mid-esophageal view; simultaneous atrial lead extraction (yellow arrow) (C), right atrial appendage prolapse into the atrial lumen (red arrow) and the separation of pericardial layers corresponding to pseudo-cardiac tamponade (blue arrow) (D). 

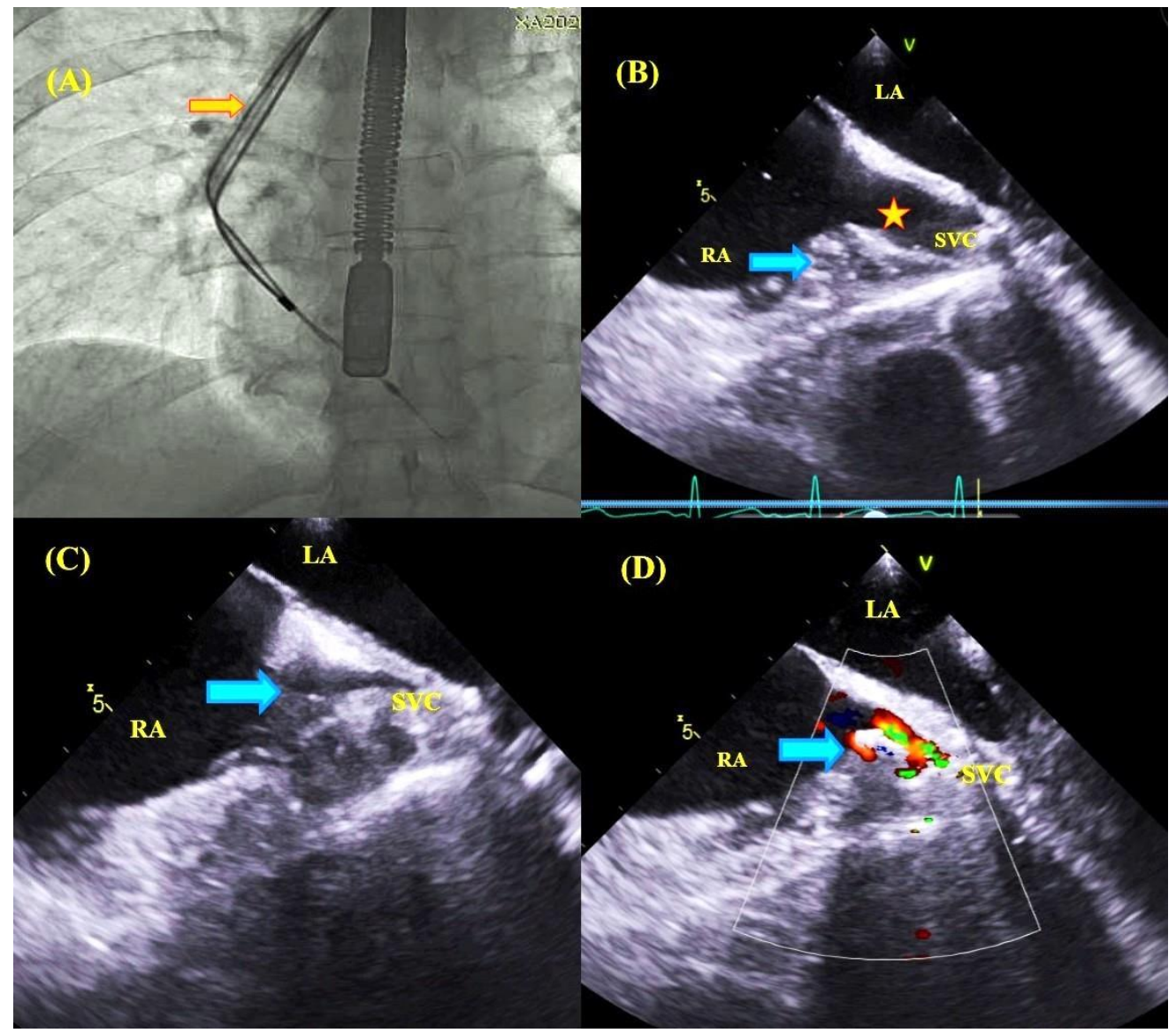

Figure 5. Comparison of fluoroscopic and TEE images during removal of lead-to-lead adhesions. Fluoroscopic image - the moment of ventricular lead extraction with simultaneous pulling on the atrial lead; the Byrd catheter slipped over the ventricular lead (orange arrow) (A). TEE images-mid-esophageal view, consecutive phases of pulling on the atrial wall and superior vena cava (blue arrows) until marked obliteration of the vessel during ventricular lead extraction and pulling on atrial lead adhesion (B-D).

Apart from providing very important information on the challenges of lead extraction, continuous TEE monitoring allows for a prompt reaction to the occurrence of directly life-threatening complications: pericardial tamponade, hemorrhagic pleural effusion and tricuspid valve injury (Figure 2). It is interesting to note that a reduction in the most severe TLE complications was encountered in patients at potentially higher procedural risk. This study shows that patients with TEE guidance had older leads and were generally at higher risk of major complications according to the SAFeTY TLE score (6.143 $\pm 4.395 \mathrm{vs}$. $5.593 \pm 4.127 ; p<0.001$ ) compared with non-TEE-guided procedures, which were characterized by more technical difficulties and prolonged operative time. Furthermore, patients with TEE guidance were older and presented with more comorbidities. Despite poorer clinical characteristics, complete procedural success was significantly higher in patients with TEE guidance $(p<0.01)$. The achievement of such a high rate of TLE effectiveness was also a result of echocardiographic navigation, because intraprocedural TEE frequently permitted continuation of the procedure in the situation of transient hemodynamic disorders (hypotension) associated with the traction of cardiac walls or superior vena cava (Figures 4 and 5). In this way, it was possible to avoid tamponade, and at the same time to maintain the high level of operator alertness. In accordance with the present results, previous studies have demonstrated a similar impact of echocardiographic monitoring on TLE effectiveness, confirming that continuous echocardiographic monitoring allows for the completion of the extraction procedure in these circumstances [16-18]. 
Furthermore, it should be underscored that TEE imaging provides a direct assessment of lead fragments that may remain after the extraction. It is of paramount importance for procedure effectiveness to visualize lead fragments or insulation remnants, which are invisible in fluoroscopy (Figure 6).

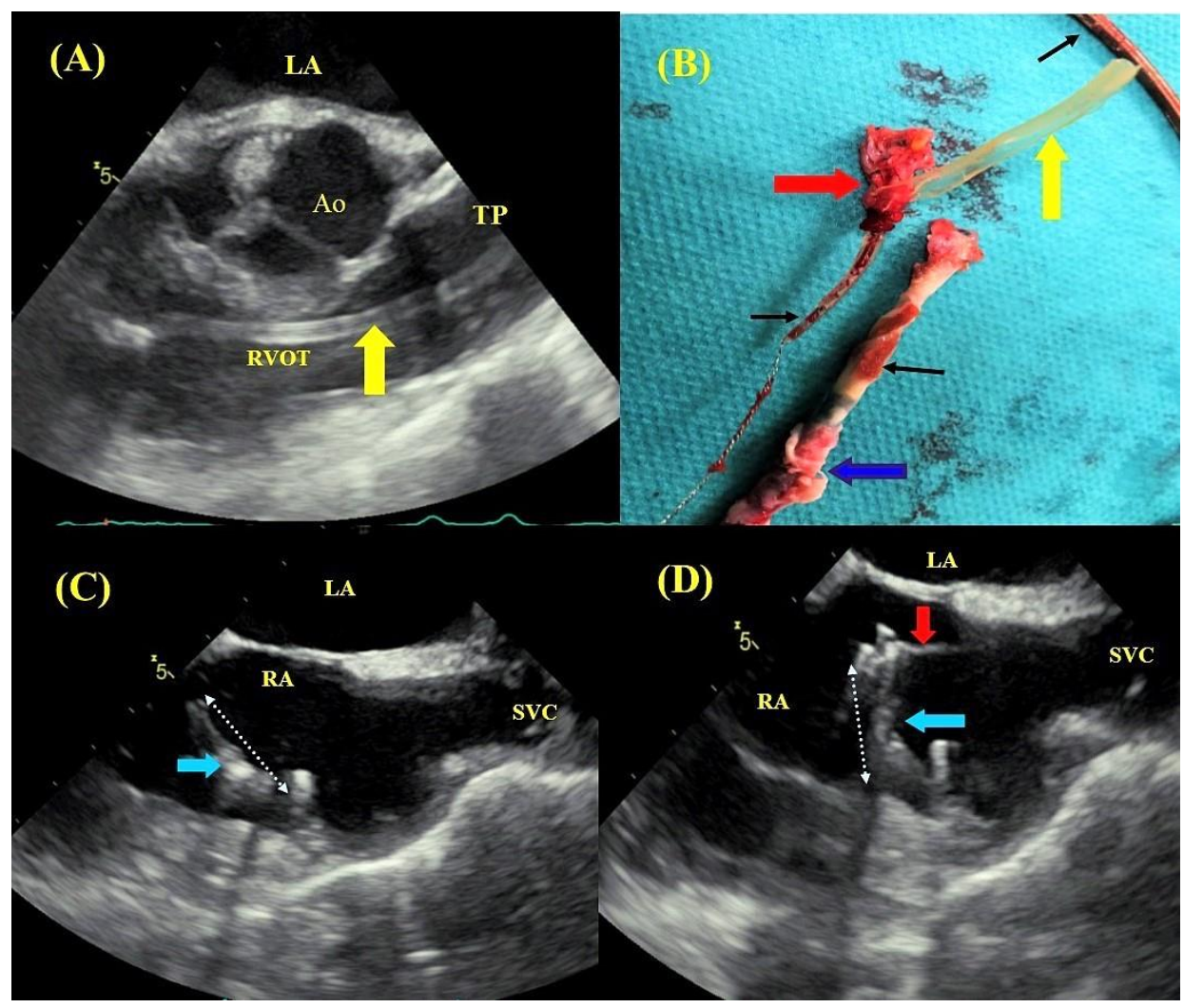

Figure 6. Remnants in heart chambers after TLE. TEE images-mid-esophageal view, transaortic view-fragment of silicone insulation (yellow arrow) stripped off the lead during TLE, dislodged into the right ventricular outflow tract (RVOT) and the pulmonary trunk (TP) (A). TEE images of the extracted ventricular lead (black arrows): unchanged fragment, lead tip with elongated guide wire, a fragment of endocardial tissue (red arrow) and silicone insulation (yellow arrow), segment of the lead surrounded by a fibrous capsule (blue arrow) and endocardial tissue (the image confirming lead adhesion to cardiac tissues) (B). TEE images—-mid-esophageal bicaval view-fragment of a thickened (hyperechogenic) atrial lead adhering to the atrial wall, fractured during TLE (white and blue arrows) (C). The free-floating tip was captured by a lasso catheter (red arrow) (D).

This is especially important for the management of patients with infective endocarditis, in whom achievement of complete procedural success is associated with a better prognosis [13].

Similarly, it is important to visualize the migration of lead adhesions and vegetation, especially to the pulmonary artery. In these situations, TEE has an unquestionable advantage over fluoroscopy and is a valuable adjunct in the procedure of lead extraction (Figure 7) [10].

At short-term follow-up (6 and 12 months) survival after TLE was comparable between the groups. This is in accordance with earlier observations, which showed dissimilarity in risk factors for complications of TLE and factors influencing short- and long-term survival [5,11,12,22]. It is well known that survival after TLE is dependent mainly on clinical variables: patient age and comorbidities: diabetes mellitus, heart failure, renal failure. As previously stated, TEE-guided patients were characterized by older age and more comorbidities (Charlson comorbidity index 4.972 vs. $4.434 ; p<0.001$ ) compared with non-TEE-guided individuals, which must have contributed to the equalization of death rates between the two groups at follow-up. 


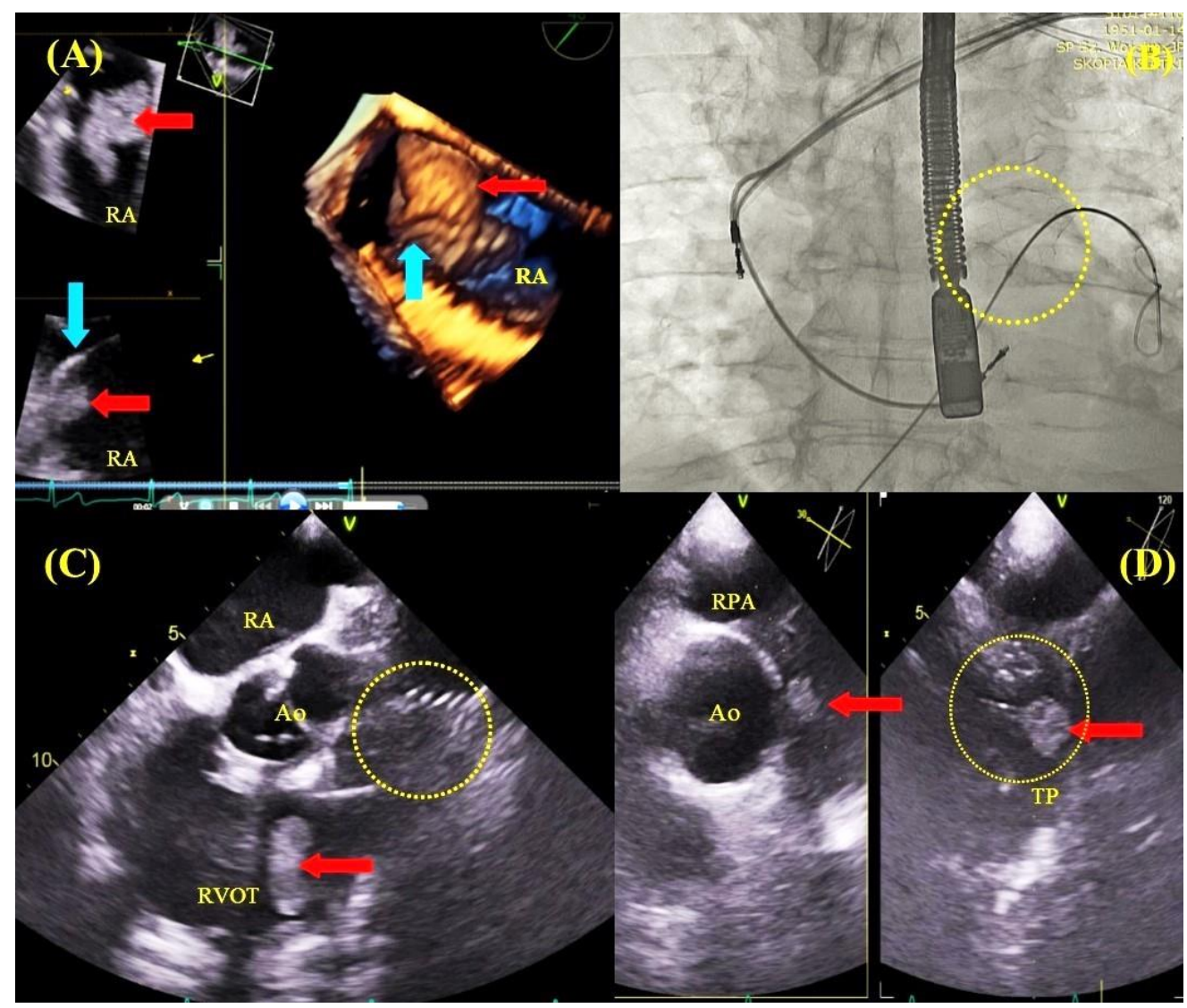

Figure 7. TLE in the course of lead-related infective endocarditis with pulmonary embolization protection devices and TEE guidance. 2D and D TEE images-a vegetation $4.0 \times 1.7 \mathrm{~cm}$ in size (red arrow) on the ventricular lead (blue arrow) in the right atrium (A). Fluoroscopy-a nitinol basket (yellow circle) positioned within the pulmonary artery (pulmonary embolization protection), pulling on both leads (lead-to-lead adhesion) during atrial lead extraction (B). TEE images-mid-esophageal view, dislodgement of the vegetation (red arrow) into the RVOT after removal of the ventricular lead (C). TEE images-upper esophageal view (Multi D imaging), vegetation fragment captured by the basket in the pulmonary artery (D). However, it should be emphasized that there were no periprocedural deaths among TEE-guided patients, whereas six patients died among the non-TEE-guided individuals. In view of the above, this is the first study to document that use of continuous TEE monitoring in patients undergoing transvenous lead extraction, and it translates directly into a reduction in periprocedural mortality.

\section{Limitations}

The organizational model of TLE procedures at the extraction centers was not the same and it did not take into account operator learning curves. Echocardiographic evaluation was not performed by one diagnostician. The study did not describe in detail the role of echocardiographic monitoring for the assessment of tricuspid valve function, as this parameter is not incorporated into the SAFeTY TLE scoring system.

\section{Conclusions}

This is the first study to confirm the higher procedural effectiveness and reduced incidence of cardiac tear and venous injury during transvenous lead extraction under transesophageal echocardiographic guidance. It was also shown that the use of continuous TEE monitoring was associated with a $100 \%$ periprocedural survival. Echocardiographic monitoring allows operators to perform many complex procedures safely and with more precision in patients at high procedural risk, through anticipating 
and thus preventing the occurrence of complications based on an analysis of changes in cardiac structures in response to the operator's maneuvers, which are invisible in fluoroscopy. Such behavior also contributes to the modification of procedures and techniques in some cases, and consequently minimizes the risk of major complications.

Author Contributions: D.N. investigation, original draft preparation; W.J. methodology, statistical study; A.P. writing, investigation; Ł.T., K.T., P.S., D.S.-S. data processing; A.T., W.B. investigation; A.K. (Andrzej Kleinrok) data processing; A.K. (Andrzej Kutarski) project manager. All authors have read and agreed to the published version of the manuscript.

Acknowledgments: We are grateful to all study participants for the use of their data. We would like to express our deepest gratitude to everyone who cooperated in the study.

Conflicts of Interest: The authors declare no conflict of interest.

\section{References}

1. Byrd, C.L.; Schwartz, S.J.; Hedin, N. Lead extraction. Indications and techniques. Cardiol. Clin. 1992, 10, 735-748. [CrossRef]

2. Byrd, C.L.; Wilkoff, B.L.; Love, C.J; Sellers, T.D.; Turk, K.T.; Reeves, R.; Young, R.; Crevey, B.; Kutalek, S.P.; Freedman, R. Intravascular extraction of problematic or infected permanent pacemaker leads: 1994-1996. U.S. Extraction Database, MED Institute. Pacing Clin. Electrophysiol. 1999, 22, 1348-1357. [CrossRef] [PubMed]

3. Raatikainen, M.J.P.; Arnar, D.O.; Merkely, B.; Nielsen, J.C.; Hindricks, G.; Heidbuchel, H.; Camm, J. A Decade of Information on the Use of Cardiac Implantable Electronic Devices and Interventional Electrophysiological Procedures in the European Society of Cardiology Countries: 2017 Report from the European Heart Rhythm Association. Europace 2017, 19, 1-90. [CrossRef] [PubMed]

4. Kennergren, C.; Bjurman, C.; Wiklund, R.; Gäbel, J. A single-centre experience of over one thousand lead extractions. Europace 2009, 11, 612-617. [CrossRef] [PubMed]

5. Wazni, O.; Epstein, L.M.; Carrillo, R.G.; Love, C.; Adler, S.W.; Riggio, D.W.; Karim, S.; Bashir, J.; Greenspon, A.J.; DiMarc, J.P.; et al. Lead extraction in the contemporary setting: The LExICon study. J. Am. Coll. Cardiol. 2010, 55, 579-586. [CrossRef]

6. Di Monaco, A.; Pelargonio, G.; Narducci, M.L.; Manzoli, L.; Boccia, S.; Flacco, M.E.; Capasso, L.; Barone, L.; Perna, F.; Bencardino, G.; et al. Safety of transvenous lead extraction according to centre volume: A systematic review and meta-analysis. Europace 2014, 16, 1496-1507. [CrossRef]

7. Poole, J.E.; Gleva, M.J.; Mela, T.; Chung, M.K.; Uslan, D.Z.; Borge, R.; Gottipaty, V.; Shinn, T.; Dan, D.; Feldman, L.A.; et al. Complication rates associated with pacemaker or implantable cardioverter defibrillator generator replacements and upgrade procedures: Results from the REPLACE registry. Circulation 2010, 122, 1553-1561. [CrossRef]

8. Barakat, A.F.; Wazni, O.M.; Tarakji, K.; Saliba, W.I; Nimri, N.; Rickard, J.; Brunner, M.; Bhargava, M.; Kanj, M.; Baranowski, B.; et al. Transvenous lead extraction at the time of cardiac implantable electronic device upgrade: Complexity, safety, and outcomes. Heart Rhythm 2017, 14, 1807-1811. [CrossRef]

9. Mazzone, P.; Migliore, F.; Bertaglia, E.; Facchin, D.; Daleffe, E.; Calzolari, V.; Crosato, M.; Melillo, F.; Peruzza, F.; Marzi, A.; et al. Safety and efficacy of the new bidirectional rotational Evolution $₫$ mechanical lead extraction sheath: Results from a multicentre Italian registry. Europace 2018, 20, 829-834. [CrossRef]

10. Bontempi, L.; Vassanelli, F.; Cerini, M.; D’Aloia, A.; Vizzardi, E.; Gargaro, A.; Chiusso, F.; Mamedouv, R.; Lipari, A.; Curnis, A. Predicting the difficulty of a lead extraction procedure: The LED index. J. Cardiovasc. Med. 2014, 15, 668-673. [CrossRef]

11. Brunner, M.P.; Yu, C.; Hussein, A.A.; Tarakji, K.G.; Wazni, O.M.; Kattan, M.W.; Wilkoff, B.L. Nomogram for predicting 30-day all-cause mortality after transvenous pacemaker and defibrillator lead extraction. Heart Rhythm 2015, 12, 2381-2386. [CrossRef] [PubMed]

12. Oszczygieł, E.; Kutarski, A.; Oszczygieł, A.; Mańkowska-Załuska, B.; Chudzik, M.; Wranicz, J.K.; Cygankiewicz, I. Risk score to assess mortality risk in patients undergoing transvenous lead extraction. Pacing Clin. Electrophysiol. 2017, 40, 918-923. [CrossRef] [PubMed]

13. Kusumoto, F.M.; Schoenfeld, M.H.; Wilkoff, B.; Berul, C.I.; Birgersdotter-Green, U.M.; Carrillo, R.; Cha, Y.M.; Clancy, J.; Deharo, J.C.; Ellenbogen, K.A.; et al. 2017 HRS expert consensus statement on cardiovascular implantable electronic device lead management and extraction. Heart Rhythm 2017, 14, e503-e551. [CrossRef] 
14. Bongiorni, M.G.; Kennergren, C.; Butter, C.; Deharo, J.C.; Kutarski, A.; Rinaldi, C.A.; Romano, S.L.; Maggioni, A.P.; Andarala, M.; Auricchio, A.; et al. The European Lead Extraction ConTRolled (ELECTRa) study: European Heart Rhythm Association (EHRA) Registry of Transvenous Lead Extraction Outcomes. Eur. Heart J. 2017, 38, 2995-3005. [CrossRef]

15. Jacheć, W.; Polewczyk, A.; Polewczyk, M.; Tomasik, A.; Kutarski, A. Transvenous Lead Extraction SAFeTY Score for Risk Stratification and Proper Patient Selection for Removal Procedures Using Mechanical Tools. J. Clin. Med. 2020, 28, 361. [CrossRef]

16. Endo, Y.; O’Mara, J.E.; Weiner, S.; Han, J.; Goldberger, M.H.; Gordon, G.M.; Nanna, M.; Ferrick, K.J.; Gross, J.N. Clinical utility of intraprocedural transesophageal echocardiography during transvenous lead extraction. J. Am. Soc. Echocardiogr. 2008, 21, 861-867. [CrossRef]

17. Hilberath, J.N.; Burrage, P.S.; Shernan, S.K.; Varelmann, D.J.; Wilusz, K.; Fox, J.A.; Eltzschig, H.K.; Epstein, L.M.; Nowak-Machen, M. Rescue transoesophageal echocardiography for refractory haemodynamic instability during transvenous lead extraction. Eur. Heart J. Cardiovasc. Imaging 2014, 15, 926-932. [CrossRef]

18. Regoli, F.; Caputo, M.; Conte, G.; Faletra, F.F.; Moccetti, T.; Pasotti, E.; Cassina, T.; Casso, G.; Schlotterbeck, H.; Engeler, A.; et al. Clinical utility of routine use of continuous transesophageal echocardiography monitoring during transvenous lead extraction procedure. Heart Rhythm 2015, 12, 313-320. [CrossRef]

19. Oestreich, B.A.; Ahlgren, B.; Seres, T.; Zipse, M.M.; Tompkins, C.; Varosy, P.D.; Aleong, R.G. Use of Transesophageal echocardiography to improve the safety of transvenous lead extraction. JACC Clin. Electrophysiol. 2015, 1, 442-448. [CrossRef]

20. Strachinaru, M.; Kievit, C.M.; Yap, S.C.; Hirsch, A.; Geleijnse, M.L.; Szili-Torok, T. Multiplane/3D transesophageal echocardiography monitoring to improve the safety and outcome of complex transvenous lead extractions. Echocardiography 2019, 36, 980-986. [CrossRef]

21. Nowosielecka, D.; Polewczyk, A.; Jacheć, W.; Tułecki, Ł.; Tomków, K.; Stefańczyk, P.; Kleinrok, A.; Kutarski, A. A new approach to the continuous monitoring of transvenous lead extraction using transesophageal echocardiography-Analysis of 936 procedures. Echocardiography 2020. [CrossRef] [PubMed]

22. Brunner, M.P.; Cronin, E.M.; Duarte, V.E.; Yu, C.; Tarakji, K.G.; Martin, D.O.; Callahan, T.; Cantillon, D.J.; Niebauer, M.J.; Saliba, W.I.; et al. Clinical predictors of adverse patient outcomes in an experience of over five thousand chronic endovascular pacemaker and defibrillator lead extractions. Heart Rhythm 2014, 11, 799-805. [CrossRef] [PubMed] 